\title{
Uma análise sobre as características da neutropenia febril: revisão de literatura
}

An analysis of the characteristics of febrile neutropenia: literature review

Un análisis de las características de la neutropenia febril: revisión de la literatura

João Pedro Franco Cerqueira ${ }^{1 *}$, Tarcila Silveira de Paula Fonseca ${ }^{1}$, Maria Thereza Castilho dos Santos ${ }^{1}$, Gabriel de Lima Machado da Fonseca ${ }^{1}$, Marianna Ramalho de Sousa ${ }^{1}$, Pedro Henrique Almeida Warol ${ }^{1}$, Emílio Conceição de Siqueira ${ }^{1}$.

\section{RESUMO}

Objetivo: Analisar as características da Neutropenia Febril (NF) em pacientes oncológicos. Revisão bibliográfica: A neutropenia febril é definida como uma temperatura maior que $38^{\circ} \mathrm{C}$ associada com uma contagem Absoluta de Neutrófilos (ANC) inferior a 500 células/microlitro. Os portadores de neoplasia hematológica têm $80 \%$ de chance de apresentar NF durante seu tratamento, enquanto, pacientes com tumores sólidos têm essa porcentagem reduzida a 30\%. Fatores como a intensidade do tratamento quimioterápico, uso de cateteres centrais e dispositivos médicos externos, fatores ambientais e geográficos, duração da internação hospitalar podem corroborar para a gravidade e duração da neutropenia. $O$ tratamento é realizado já na primeira hora com antibioticoterapia de amplo espectro para organismos grampositivos e gram-negativos. Considerações finais: A neutropenia febril é uma grave consequência do tratamento de neoplasias, pois significa um atraso na terapêutica do câncer além dos grandes custos ao sistema de saúde e de representar risco de vida ao paciente. Nesse contexto, torna-se essencial o treinamento dos profissionais de saúde para esta patologia além de uma ação imediata com a introdução de antibioticoterapia e realização da pesquisa do sítio de infecção, reduzindo, assim, sua morbimortalidade.

Palavras-chave: Neutropenia febril, Neutropenia febril induzida por quimioterapia, Oncologia.

\begin{abstract}
Objective: To analyze the characteristics of Febrile Neutropenia (NF) in cancer patients. Bibliographic review: Febrile neutropenia is defined as a temperature greater than $38^{\circ} \mathrm{C}$ associated with an Absolute Neutrophil Count (ANC) of less than 500 cells/microliter. Patients with hematological malignancy have an $80 \%$ chance of having NF during their treatment, while patients with solid tumors have this percentage reduced to $30 \%$. Factors such as the intensity of chemotherapy treatment, use of central catheters and external medical devices, environmental and geographical factors, length of hospital stay may corroborate the severity and duration of neutropenia. Treatment is performed within the first hour with broad-spectrum antibiotic therapy for gram-positive and gram-negative organisms. Final considerations: Febrile neutropenia is a serious consequence of the treatment of neoplasms, as it means a delay in cancer therapy in addition to the great costs to the health system and representing a risk to the patient's life. In this context, it is essential to train health professionals for this pathology, in addition to taking immediate action with the introduction of antibiotic therapy and carrying out the investigation of the infection site, thus reducing its morbidity and mortality.
\end{abstract}

Keywords: Febrile neutropenia, Chemotherapy-induced febrile neutropenia, Medical oncology.

${ }^{1}$ Universidade de Vassouras (UV), Vassouras - RJ. *E-mail: joão.lazarone1997@gmail.com

SUBMETIDO EM: 2/2022

ACEITO EM: 2/2022

PUBLICADO EM: 3/2022 


\section{RESUMEN}

Objetivo: Analizar las características de la Neutropenia Febril (NF) en pacientes oncológicos. Revisión bibliográfica: La neutropenia febril se define como una temperatura superior a $38{ }^{\circ} \mathrm{C}$ asociada con un Recuento Absoluto de Neutrófilos (RAN) inferior a 500 células/microlitro. Los pacientes con neoplasia hematológica tienen un $80 \%$ de probabilidad de tener NF durante su tratamiento, mientras que los pacientes con tumores sólidos tienen este porcentaje reducido al $30 \%$. Factores como la intensidad del tratamiento de quimioterapia, el uso de catéteres centrales y dispositivos médicos externos, factores ambientales y geográficos, la duración de la estancia hospitalaria pueden corroborar la gravedad y la duración de la neutropenia. El tratamiento se realiza dentro de la primera hora con terapia antibiótica de amplio espectro para organismos grampositivos y gramnegativos. Consideraciones finales: La neutropenia febril es una consecuencia grave del tratamiento de las neoplasias, ya que implica un retraso en la terapia del cáncer además de los grandes costos para el sistema de salud y representa un riesgo para la vida del paciente. En este contexto, es fundamental capacitar a los profesionales de la salud para esta patología, además de actuar de inmediato con la instauración de la antibioticoterapia y realizar la investigación del sitio de infección, reduciendo así su morbimortalidad.

Palavras clave: Neutropenia febril, Neutropenia febril inducida por quimioterapia, Oncología médica.

\section{INTRODUÇÃO}

O tratamento de pacientes com neoplasias é dependente de fatores que vão desde a confirmação diagnóstica da doença até o estadiamento e às características individuais e mentais/emocionais do indivíduo. Nesse contexto, existem várias abordagens terapêuticas: hormonioterapia, procedimentos cirúrgicos, radioterapia e quimioterapia (FERREIRA JN, et al., 2017; HANSEN BA, et al., 2020).

A quimioterapia antineoplásica é uma opção terapêutica muitas vezes indispensável no tratamento do câncer. Os quimioterápicos atuam em células tumorais e também em células distintas do organismo, como medula óssea, cabelo e mucosa do trato digestivo, sendo aplicada em ciclos repetitivos porque cada célula normal, apresenta um período de recuperação (FERREIRA JN, et al., 2017; HANSEN BA, et al., 2020).

A duração da exposição tóxica aos medicamentos depende da quantidade utilizada, da quantidade utilizada para o tratamento e das características inerentes do paciente. As drogas podem ocasionar toxicidade e efeitos indesejáveis, tais quais como alopecia, mielodepressão e febre. A ocorrência de febre em pacientes em tratamento quimioterápico representa uma emergência oncológica, pois indica neutropenia febril (NF), a qual representa uma grave complicação altos níveis de mortalidade que podem chegar a mais de 50\% (FERREIRA JN, et al., 2017; STERN A, et al., 2019).

Neutropenia Febril (NF) é definida como uma contagem absoluta de neutrófilos menos de 500 células por milímetro cúbico e uma temperatura igual ou superior a $38,3^{\circ} \mathrm{C}$ isoladamente, ou superior a $38^{\circ} \mathrm{C}$ continuamente, durante uma hora. Na presença de neutropenia febril deve-se suspeitar de um possível foco de infecção bacteriana (ARROYAVE-PEÑA T, et al., 2019; PIRES MBT, MAMPRIM G., 2021).

O risco de desenvolver infecções mais graves e complicadas está claramente ligado ao grau e duração da neutropenia. O risco de infecções graves não é uniforme entre esses pacientes, e os fatores associados à maior suscetibilidade a complicações infecciosas incluem neutropenia prolongada, uso de quimioterapia de resgate e recidiva da doença. Entretanto, outros fatores além da própria leucopenia estão associados ao risco infeccioso (HANSEN BA, et al., 2020; COOKSLEY T, et al., 2021).

O início imediato da antibioticoterapia empírica em pacientes com NF é o padrão de atendimento e visa prevenir a morte precoce (STERN A, et al., 2019; GUARANA M, et al., 2019). O quadroinfeciosopodeevoluir de forma rápida com instabilidade hemodinâmica, sendo recomendado o início de terapêutica antibiótica até 1 hora após a documentação de febre (ATALAIA G, et al., 2015).

A NF ocasiona, frequentemente, a reduções de dose e adiamentos de tratamento, comprometendo o prognóstico de doença oncológica potencialmente curável (ATALAIA G, et al., 2015). Nesse sentido, torna- 
se essencial o conhecimento dos profissionais de saúde acerca dessa complicação tão comum no tratamento das neoplasias a fim de propiciar a percepção e o tratamento precoce para reduzir a morbimortalidade. $\mathrm{O}$ objetivo do estudo foi analisar as características da neutropenia febril em pacientes oncológicos.

\section{REVISÃO BIBLIOGRÁFICA}

\section{Definição}

A neutropenia febril é definida como uma temperatura maior que $38^{\circ} \mathrm{C}$ associada com uma contagem absoluta de neutrófilos (ANC) inferior a 500 células/microlitro (menos de $0,5 \times 10^{9} / \mathrm{L}$ ). Este ponto de corte de neutrófilos foi escolhido porque o risco de sepse avassaladora aumenta à medida que a CAN cai abaixo de $0,5 \times 10^{9} / L$ (DAVIS K e WILSON S, 2020; FERREIRA JN, et al., 2017).

\section{Epidemiologia}

As infecções são as causas mais comuns de morte em pacientes com câncer como resultado da terapia. O risco de NF ou infecções com risco de vida correlaciona-se com a gravidade e duração da neutropenia. O risco de infecção aumenta significativamente abaixo de 1.000 neutrófilos por microlitro (LINK $\mathrm{H}, 2019$ ).

Os portadores de neoplasia hematológica têm $80 \%$ de chance de apresentar NF durante seu tratamento, enquanto, pacientes com tumores sólidos têm essa porcentagem reduzida a $30 \%$. Fatores como a intensidade do tratamento quimioterápico, uso de cateteres centrais e dispositivos médicos externos, fatores ambientais e geográficos, duração da internação hospitalar podem corroborar para a gravidade e duração da neutropenia (ATALAIA G, et al., 2015).

O prognóstico é pior em pessoas com bacteremia comprovada, com taxas de mortalidade de $18 \%$ e $5 \%$ relatadas em pessoas com bacteremia Gram-negativa e Gram-positiva, respectivamente. Os idosos estão em maior risco de neutropenia febril após quimioterapia, com piores taxas de morbidade e mortalidade (MHASKAR R, et al.,2015; HOSONO Y, et al., 2018).

Em muitos pacientes com neutropenia febril não é possível encontrar um foco infeccioso, uma vez que este é apenas detectável em 20\% a 30\% e em hemoculturas tomadas (ARROYAVE-PEÑA T, et al., 2019). Estudos em doentes oncológicos com NF reportaram mortalidades na ordem de 5 a $20 \%$, aumentando de forma diretamente proporcional ao número de comorbidades médicas e complicações infecciosas do doente (ATALAIA G, et al., 2015).

A incidência global da NF é difícil de avaliar, pois os eventos nem sempre são identificados ou relatados. As internações neutropênicas, frequentemente associadas à NF, são mais simples de quantificar. Estudos dos Estados Unidos da América (EUA) sugerem uma estimativa de 7,83 hospitalizações em decorrência de neutropenia por 1.000 pacientes com câncer a cada ano (60.294 casos em 1999), com incidência aumentando para 43,3 casos por 1.000 pacientes naqueles com neoplasias hematológicas. Evidências de ensaios clínicos e prática clínica sugerem que a incidência de NF pode ser ainda maior (até 117 casos por 1.000 pacientes) (AAPRO M, et al., 2017).

Estima-se que nos Estados Unidos mais de 60.000 pessoas com câncer são hospitalizadas com neutropenia e mais 4.000 pessoas morrem de neutropenia febril a cada ano. Com uma taxa de mortalidade variando entre $2 \%$ e $21 \%$ entre pacientes hospitalizados por complicações de neutropenia febril, a neutropenia relacionada ao câncer é uma causa substancial de mortalidade evitável entre pacientes com neoplasias (TAI E, et al., 2017).

\section{Classificação da neutropenia febril}

Indivíduos com NF podem ser classificados como neutropênicos de baixo risco, risco intermediário e alto risco. O escore de risco é determinado pelo índice de risco Associação Multinacional para Cuidados de Suporte ao Câncer (MASCC), que atribui pontos de acordo com a importância de cada variável: paciente assintomático; paciente apresentando sintomas leves, moderados ou graves; sem hipotensão; sem doença 
pulmonar obstrutiva crônica; tumor sólido sem infecção fúngica prévia; sem desidratação; estado ambulatorial no início da febre; e idade inferior a 60 anos. O índice de risco MASCC tem pontuação máxima de 26 pontos e classifica os pacientes em baixo risco ( $\geq 21$ pontos) ou alto risco (<21 pontos). É amplamente utilizado e considerado simples, apresentando boa sensibilidade e alto valor positivo (FERREIRA JN, et al., 2017).

A quimioterapia citotóxica causa neutropenia por supressão transitória da hematopoiese da medula óssea. A maioria dos regimes de quimioterapia está associada a 6-8 dias de neutropenia, com incidência de NF de 8 episódios por 1.000 pacientes. Existem diferentes incidências de NF, atualmente classificadas como regimes de baixo (ou seja, em $<10 \%$ dos tratamentos quimioterápicos), intermediário (em 10-20\%) e alto risco (em >20\%). No grupo de NF de alto risco, o uso profilático do fator estimulador de colônias de granulócitos (G-CSF) é recomendado pelas diretrizes da prática clínica (MOREIRA-PINTO J, et al., 2020).

\section{Etiologia}

Muitas substâncias citotóxicas prejudicam a formação de células sanguíneas a partir de células-tronco hematopoiéticas pluripotentes na medula óssea e, em particular, a granulopoiese a partir da qual os granulócitos neutrófilos se desenvolvem. Como a toxicidade limitante de dose mais importante, a neutropenia, a neutropenia febril e as infecções podem comprometer o sucesso da quimioterapia pelo risco de morte e pela resultante redução da intensidade da dose da quimioterapia (LINK H, 2019).

Pacientes com neutropenia grave não apresentam os sinais inflamatórios típicos de uma infecção devido à deficiência de neutrófilos. A febre é, portanto, o primeiro e muitas vezes o único sinal de infecção. Como os pacientes são imunossuprimidos em decorrência da quimioterapia, uma infecção se espalha no organismo em poucas horas e pode levar à morte (LINK H, 2019).

Pacientes que esperavam neutropenia por menos de sete dias e sem comorbidades são considerados de baixo risco. Neste grupo estão os pacientes tratados para tumores sólidos nos quais os regimes de quimioterapia não produzem neutropenia tão grave e prolongada como quimioterapia intensificada para 0 tratamento de certas malignidades hematológicas. Pacientes que esperavam supressão da medula óssea à longo prazo (neutropenia grave por mais de sete dias) e/ou comorbidades médicas significativa apresentam alto risco de complicações graves relacionadas à infecção e superinfecções por bactérias secundárias, micoses invasivas e vírus (JOHANSSON S e JENSEN LH, 2015).

Entre os determinantes mais importantes do risco de NF estão o tipo de quimioterapia e a intensidade da dose. O risco de NF na maioria dos regimes de terapia para o tratamento de vários tumores frequentes é maior durante os primeiros ciclos de terapia do que nos ciclos subsequentes. Isso pode ser explicado pelo fato de que, nos ciclos seguintes, muitas vezes são utilizados fatores de crescimento hematopoiéticos e a dose de quimioterapia é reduzida. Sem esse ajuste, o risco de FN durante todos os ciclos é constante. Se ocorrerem complicações neutropênicas, um alto risco de NF também pode ser assumido no ciclo subsequente (LINK H, 2019).

A patologia da FN é multifatorial. Os fatores contribuintes incluem:pancitopenia, substituição da medula, defeitos qualitativos da imunidade humoral e celular,mucosite e infecção por cateter venoso central (CVC)(DAVIS K e WILSON S, 2020).

A infecção bacteriana é a complicação maus comum associada à neutropenia induzida por quimioterapia, e a maioria das infecções microbiologicamente comprovadas originam-se da microflora endógena. Até 1980, bactérias gram-negativas especialmente a Escherichia coli e Pseudomonas aeruginosa eram os patógenos mais frequentemente isolados em pacientes com neutropenia. Atualmente, as bactérias gram-positivas compõem até $70 \%$ de todas as infecções neutropênicas com os isolados mais comuns sendo Staphylococcusepidermidis endógeno, Staphylococcus aureus e Streptococcus. Essa mudança é resultado do uso de antibioticoterapia profilática contra bactérias gram-negativas, da intensificação da incidência de mucosite oral e ao aumento do consumo de cateteres venosos centrais. No entanto, a tendência para bactérias gram-negativas resistentes levou novamente a um incremento nas infecções principalmente por Escherichia coli endógena (JOHANSSON S e JENSEN LH, 2015). 


\section{Diagnóstico}

A história e um exame completos são recomendados para determinar a fonte de infecção. A febre pode ser o único sinal de infecção na neutropenia. Os pacientes podem apresentar hipotermia, especialmente no cenário de infecção grave (LONG B, et al., 2021). Os focos infecciosos mais comuns foram pele e trato gastrointestinal, incluindo também o trato respiratório (ARROYAVE-PEÑA T, et al., 2019; GOLDSMITH C, et al., 2018).

Devido à redução da resposta inflamatória, os pacientes muitas vezes não desenvolvem os achados clássicos de infecção. Por exemplo, aqueles com celulite podem ter eritema sutil, enquanto aqueles com pneumonia podem ter sintomas respiratórios leves e uma radiografia de tórax negativa. A cavidade oral deve ser examinada para mucosite, o que aumenta o risco de infecção e desfechos ruins (LONG B, et al., 2021).

Um exame físico completo é crucial em todos os pacientes com NF e deve ser repetido pelo menos diariamente enquanto o paciente não estiver bem. Um foco de infecção muitas vezes não é encontrado, porque a falta de neutrófilos prejudica a capacidade do sistema imunológico de produzir pus, eritema e dor localizada. Portanto, a revisão regular repetida é essencial e os sinais sutis não devem ser ignorados ou descartados como irrelevantes (BA Y, et al., 2020; DAVIS K e WILSON S, 2020).

O exame físico deve incluir, mas não se limitar a: sinais vitais (temperatura, frequência respiratória, pressão arterial e frequência cardíaca) e avaliação de instabilidade hemodinâmica, temperatura oral ou timpânica. A temperatura retal não deve ser medida em pacientes oncológicos porque a integridade da mucosa retal comprometida pode induzir uma bacteremia Gram-negativa mesmo com trauma leve (DAVIS K e WILSON S, 2020; GOLDSMITH C, et al., 2018).

Sinais cutâneos de celulite, abscesso ou ruptura iminente da integridade da pele, inclusive ao redor do CVC (se houver), períneo e dobras cutâneas labiais; exame da boca para mucosite ou gengivite; exame dos ouvidos, nariz e garganta em busca de sinais de infecção do trato respiratório superior, otite média e sinusite. Além disso, é essencial um exame respiratório cuidadoso, sinais mais sutis de infecção podem incluir apenas uma frequência respiratória elevada. Deve-se auscultar o coração e realizar um exame abdominal incluindo a procura de fissura anal, além de verificar se há hepatoesplenomegalia (DAVIS $\mathrm{Ke}$ WILSON S, 2020; LONG B, et al., 2021).

Deve-se realizar hemocultura ainda na primeira hora, mas esta não deve atrasar o início da antibioticoterapia. Ademais, deve-se fazer exames de sangue de rotina, incluindo hemograma completo, testes de função renal e hepática, marcadores de inflamação como Proteína C-Reativa(PCR), Velocidade de Hemossedimentação(VHS) e/ouprocalcitoninapodem ser considerados, mas podem ser falsamente tranqüilizadores. Pode-se considerar uma gasometria para lactato venoso se o paciente não estiver bem para avaliar sepse (DAVIS K e WILSON S, 2020; CERCHIONE C, et al., 2021).

\section{Neutropenia febril em crianças}

Crianças com câncer tratadas com drogas antineoplásicas/citotóxicas apresentam neutropenia como consequência direta de seu câncer. Aproximadamente um terço dessas crianças desenvolve febre durante 0 período neutropênico, ou três episódios de neutropenia febril por ano (RIVERA-SALGADO D, et al., 2018; TRIFILIO S e MEHTA J, 2019).

A mortalidadeassociada à neutropenia febril em pediatria é de cerca de $2-3 \%$ se os pacientes recebem tratamento antimicrobiano, enquanto mortalidade de até $50 \%$ foi relatada em pacientes que não receberam antibioticoterapia nas primeiras 48 horas após o início do quadro. Os dados de mortalidade nos EUA são muito semelhantes aos relatados na América Latina, sendo a maioria dos estudos provenientes do Chile e da Argentina (RIVERA-SALGADO D, et al., 2018).

\section{Manejo de pacientes com neutropenia febril na pandemia de COVID-19}

Todos os pacientes com neutropenia febril estarão em risco e potencialmente já com o novo coronavírus (COVID-19) positivos. Apesar da ausência de um alto nível de evidência, em casos de neutropenia febril, 
uma Tomografia Computadorizada (TC) de tórax deve ser realizada sistematicamente mesmo na ausência de tosse. Essa prática seria justificada pelos dados de um estudo que mostrou que as lesões tomográficas de COVID foram mais frequentes em pacientes com câncer. Testes biológicos baseados na reação em cadeia da polimerase podem ser feitos dependendo da disponibilidade e dos resultados da tomografia computadorizada. Essa triagem sistemática de COVID-19 entre neutropenia febril pode reduzir o contágio intra-hospitalar e permitir o tratamento precoce (BOUTAYEB S, et al., 2020; PALUKURI NR, et al., 2020).

\section{Tratamento}

A febre na neutropenia deve sempre ser considerada uma infecção com risco de vida, que deve ser tratada dentro de $1 \mathrm{~h}$ com antibióticos de forma padronizada além de medidas de suporte básico de vida. Os diagnósticos necessários não devem atrasar a terapia (LINK H, 2019; LONG B, et al., 2021; TRIFILIO S e MEHTA J, 2019).

A terapia empírica depende dos patógenos encontrados no caso de infecção comprovada, dos resultados dos estudos e da situação atual do patógeno e da resistência, bem como do aumento da multirresistência. Em princípio, os patógenos Gram-negativos e Gram-positivos relevantes devem ser cobertos (LINK H, 2019; LONG B, et al., 2021). Não há consenso na literatura sobre qual antibiótico ou combinação de antibióticos é ideal para o tratamento da NF (WANG L, et al., 2015; HOLUBEC L, et al., 2017).

O tratamento deve ser bactericida e abrangente contra os microrganismos que frequentemente desencadeiam a infecção. Como mencionado anteriormente, apenas um agente específico é detectado em aprox. Um quinto de todos os casos de neutropenia febril, portanto, embora as hemoculturas apresentem resultados negativos, o tratamento com antibióticos empíricos é considerado necessário para lidar com potenciais infecções ocultas e riscos de múltiplos patógenos (JOHANSSON S e JENSEN LH, 2015; HOLUBEC L, et al., 2017).

Embora as bactérias gram-positivas causem mais frequentemente infecção em pacientes com neutropenia, é essencial que o tratamento cubra uma ampla gama de bactérias gram-negativas devido à sua virulência e associação com síndromes de sepse grave. A dosagem do antibiótico é ajustada de acordo com a função renal (JOHANSSON S e JENSEN LH, 2015; HOLUBEC L, et al., 2017).

As diretrizes sugerem iniciar a cobertura antibiótica com um antibiótico $\beta$-lactâmico de amplo espectro como a base do tratamento inicial para NF. Aztreonam, um antibiótico monobactâmico, não tem sensibilidade cruzada com antibióticos $\beta$-lactâmicos e é uma alternativa para aqueles com alergia ou intolerância a antibióticos $\beta$-lactâmicos. As diretrizes atuais recomendam administrar Aztreonam com Vancomicina (AV) para fornecer cobertura adequada contra organismos gram-positivos e gramnegativos. Aztreonam mais vancomicina tem sido usado com sucesso para tratar neutropenia febril em pacientes com câncer (TRIFILIO S e MEHTA J, 2019).

\section{Profilaxia da neutropenia febril}

A formação de granulócitos é controlada por fatores de crescimento hematopoiéticos, como fator estimulador de colônias de granulócitos (G-CSF) ou fator estimulador de colônias de granulócitos/macrófagos (GM-CSF), que são liberados na corrente sanguínea à medida que a concentração de granulócitos diminui para estimular a proliferação ou diferenciação de células progenitoras. O G-CSF foi descrito e caracterizado bioquimicamente no início dos anos 80; o gene correspondente foi clonado e desenvolvido como uma molécula recombinante para aplicaçãoclínicaem neutropenia(LINK H, 2019; BOUTAYEB S, et al., 2020).

A administração profilática de preparações recombinantes de G-CSF, como filgrastim, lenograstim, pegfilgrastim ou lipegfilgrastim, acelera a regeneração das contagens de células de granulócitos protetoras após quimioterapia mielossupressora. Muitos ensaios clínicos estabeleceram a utilidade clínica da terapia profilática com G-CSF após quimioterapia para encurtar a neutropenia. Foi demonstrado de forma convincente que a taxa de $\mathrm{FN}$, infecções durante a neutropenia e mortalidade relacionada à neutropenia podem ser significativamente reduzidas pelouso de G-CSF (LINK H, 2019; LONG B, et al., 2021). 
Com um risco de NF de 10-20\%, a profilaxia com G-CSF só é indicada em caso de fatores de risco adicionais. A idade é um fator de risco significativo, de modo que a profilaxia com G-CSF é sempre recomendada para pacientes com idade $\geq 65$ anos se o risco de NF for superior a $10 \%$. Outros fatores de risco são: status de desempenho ruim (Eastern Cooperative Oncology Group (ECOG) > 1), comorbidade como diabetes e hipertensão, histórico de quimioterapia anterior, anemia (hemoglobina< $12 \mathrm{~g} / \mathrm{dl}$ ), linfocitopenia $(<700 / \mu \mathrm{l})$, hipoalbuminemia $(<35 \mathrm{~g} / \mathrm{dl})$ e hiperbilirrubinemia. Além disso, radioterapia prévia parece aumentar o risco de NF (LINK H, 2019; MHASKAR R, et al.,2014).

\section{Consequências da neutropenia febril}

Não apenas o aumento dos custos, mas também o comprometimento da qualidade de vida são outras consequências negativas das complicações da neutropenia. Muitos protocolos de terapia só podem atingir a intensidade de dose padrão necessária se a neutropenia e a NF puderem ser evitadas ou mantidas dentro de uma faixa clinicamente aceitável (LINK H, 2019).

\section{CONSIDERAÇÕES FINAIS}

A neutropenia febril se caracteriza pela presence de febre e diminuição dos neutrófilos em um paciente oncológico é representa uma grave consequência do tratamento de neoplasias, pois significa um atraso terapêutica do câncer além dos grandes custos ao sistema de saúde e de representar risco de vida ao paciente. Nesse contexto, torna-se essencial o treinamento dos profissionais de saúde para esta patologia além de uma ação imediata com a introdução de antibioticoterapia e realização da pesquisa do sítio de infecção, reduzindo, assim, sua morbimortalidade.

\section{REFERÊNCIAS}

1. AAPRO M, et al. Refiningthe role ofpegfilgrastim (a long-acting G-CSF) for prevention of chemotherapy-induced febrile neutropenia: consensus guidancere commendations. Support Care Cancer, 2017; 25(11): 3295-3304.

2. ARROYAVE-PEÑA T, et al. Características de los pacientes adultos con neutropenia febril enun hospital universitario (Medellín, 2012-2016). MEDICINA U.P.B., 2019; 38(2): 108-113.

3. AtAlaiA G, et al. Neutropenia Febril. Rev Clin Hosp Prof Dr Fernando Fonseca, 2015; 3(1): 13-19.

4. BA $Y$, et al. Current management ofchemotherapy-induced neutropenia in adults: key points and new challenges: Committee of Neoplastic Supportive-Care (CONS), China Anti-Cancer Association Committee of Clinical Chemotherapy, China Anti-Cancer Association. Cancer Biol Med, 2020; 17(4): 896-909.

5. BOUTAYEB S, et al. HowtoManageFebrile Neutropenia Duringthe COVID Pandemic? Oncologist. 2020; 25(8): e1251.

6. CERCHIONE C, et al. Pegfilgrastim for primary prophylaxis of febrile neutropenia in multiplemyeloma. Support Care Cancer, 2021; 29(11): 6973-6980.

7. COOKSLEY T, et al. Emerging challenges in the evaluation of fever in cancer patients at risk of febrile neutropenia in the era of COVID-19: a MASCC position paper. Support CareCancer, 2021; 29(2): 1129-1138.

8. DAVIS K, WILSON S. Febrile neutropenia in paediatriconcology. PaediatrChild Health (Oxford), 2020; 30(3): 93-97.

9. FERREIRA JN. Managingfebrile neutropenia in adult câncer patients: anintegrative review oftheliterature. RevBrasEnferm, 2017; 70(6): 1301-1308.

10. GOLDSMITH C, et al. Assessment of InitialFebrile Neutropenia Management in HospitalizedCancerPatientsat a Community Cancer Center. J AdvPractOncol, 2018; 9(6): 659-664.

11. GUARANA M, et al. Shockand Early Death in HematologicPatientswithFebrile Neutropenia. AntimicrobAgentsChemother, 2019; 63(11): e01250-19.

12. HANSEN BA, et al. Febrile Neutropenia in AcuteLeukemia. Epidemiology, Etiology, Pathophysiology and Treatment. Mediterr J HematollnfectDis, 2020; 12(1): e2020009.

13. HOLUBEC L, et al. The Effectiveness of Febrile Neutropenia Prophylaxis with Lipegfilgrastim in Routine Clinical Practice. In Vivo, 2017; 31(3): 303-306.

14. HOSONO Y, et al. Febrile neutropenia withbacterialparonychia. Clin Case Rep, 2018; 6(3): 543-544.

15. JOHANSSON S, JENSEN LH. Hurtig identification of patientermed neutropeniogfeberbe drerprognosen [Fast identification of febrile neutropenia improves theoutcome]. Ugeskr Laeger, 2015; 177(36): V03150240.

16. Link H. Hematopoietic Growth Factors in the Management of Anemia and Febrile Neutropenia. BreastCare (Basel), 2019; 14(2): 93-98. 
17. LONG B, et al. Just thefacts: febrile neutropenia in the emergency department setting. CJEM, 2021; 23(4): 445-449.

18. MHASKAR R, et al. Colony-stimulatingfactors for chemotherapy-induced febrile neutropenia. Cochrane Database Syst Rev, 2014; 2014(10): CD003039.

19. MOREIRA-PINTO J, et al. Febrile Neutropenia in Patients with Solid Tumors Under going Intravenous Chemotherapy. Oncol Res Treat, 2020; 43(11): 605-612.

20. NUCCI M. How I TreatFebrile Neutropenia. Mediterr J HematollnfectDis, 2021; 13(1): e2021025.

21. PALUKURI NR, et al. Incidence of febrile neutropenia with common lyused chemotherapy regimen in localized breast cancer. South Asian J Cancer, 2020; 9(1): 4-6.

22. PIRES MBT, MAMPRIM G. Neutropenia Febriç em pacientes com cancer de mama: revisão de ensaios clínicos. International Journal of Health Management Review, 2021; 7(1): 1-9.

23. RIVERA-SALGADO D, et al. Neutropenia febril enniñosconcáncer: manejo enelservicio de emergencias. Rev Chilena Infectol, 2018; 35(1): 62-71.

24. STERN A, et al. Early discontinuation of antibiotics for febrile neutropenia versus continuation until neutropenia resolution in people with cancer. Cochrane DatabaseSystRev, 2019; 1(1): CD012184.

25. TAI E, et al. CostofCancer-Related Neutropenia orFeverHospitalizations, United States, 2012. J Oncol Pract, 2017; 13(6): e552-e561.

26. TRIFILIO S, MEHTA J. Aztreonam and Vancomycin for Initial Treatment of Febrile Neutropenia in Penicillin-Allergic Patients During Hematopoietic Stem Cell Transplantation. J AdvPractOncol, 2019; 10(7): 685-690.

27. WANG L, et al. The impactof primary prophylaxis with granulocytecolony-stimulatingfactors on febrile neutropenia during hemotherapy: a systematic review and meta-analysisofrandomizedcontrolledtrials. SupportCareCancer, 2015; 23(11): 3131-40. 